Research Paper

\title{
Inflammatory and Senescent Phenotype of Pancreatic Stellate Cells Induced by Sqstm1 Downregulation Facilitates Pancreatic Cancer Progression
}

\author{
Chuxiao Shao ${ }^{* \bowtie 凶}$, Chaoyong $\mathrm{Tu}^{1^{*}}$, Xiangdong Cheng ${ }^{2, *}$, Zhiyuan $\mathrm{Xu}^{2}$, Xiaoguang Wang ${ }^{3}$, Jian Shen ${ }^{4}$, Kequn \\ Chai ${ }^{5,6}$, Wei Chen ${ }^{5,6}$
}

1. Department of Hepatopancreatobiliary Surgery, the Fifth Affiliated Hospital of Wenzhou Medical University, Lishui Hospital of Zhejiang University, School of Medicine, Lishui, Zhejiang 323000, P.R. China

2. Department of Gastrointestinal Surgery, The First Affiliated Hospital of Zhejiang Chinese Medicine University, Hangzhou, Zhejiang, China

3. Department of Hepatopancreatobiliary Surgery, Jiaxing Second Hospital, Jiaxing, Zhejiang, China

4. Department of Surgery, the Second Affiliated Hospital of Zhejiang University, Hangzhou, Zhejiang, China

5. Cancer Institute of Integrated traditional Chinese and Western Medicine, Key laboratory of cancer prevention and therapy combining traditional Chinese and Western Medicine, Zhejiang Academy of Traditional Chinese Medicine, Hangzhou, Zhejiang, 310012, China

6. Department of Medical Oncology, Tongde hospital of Zhejiang Province, Hangzhou, Zhejiang, 310012, China

*These authors contributed equally to this work.

$\square$ Corresponding authors: Wei Chen, Cancer Institute of Integrated traditional Chinese and Western Medicine, Zhejiang Academy of Traditional Chinese Medicine, Tongde hospital of Zhejiang Province, Hangzhou, Zhejiang, 310012, China; Email: wei chen@zju.edu.cn; Kequn Chai, Cancer Institute of Integrated traditional Chinese and Western Medicine, Zhejiang Academy of Traditional Chinese Medicine, Tongde hospital of Zhejiang Province, Hangzhou, Zhejiang, 310012, China; Email: ckq3301@aliyun.com; Chuxiao Shao, Department of Hepatopancreatobiliary Surgery, the Fifth Affiliated Hospital of Wenzhou Medical University, Lishui Hospital of Zhejiang University, School of Medical, Lishui, Zhejiang 323000, P.R. China; Email: scx1818@126.com

(c) Ivyspring International Publisher. This is an open access article distributed under the terms of the Creative Commons Attribution (CC BY-NC) license (https://creativecommons.org/licenses/by-nc/4.0/). See http://ivyspring.com/terms for full terms and conditions.

Received: 2018.06.12; Accepted: 2019.02.18; Published: 2019.04.21

\begin{abstract}
Pancreatic ductal adenocarcinoma (PDAC) has unique microenvironment with extensive infiltration of fibroblasts, which are mainly derived from the resident pancreatic stellate cells (PaSCs). As activated PaSCs constitute a major contributor to pancreatic cancer progression, the mechanisms underlying their activation have been being intensively studied. Previous studies showed that Sequestosome-1 (sqstml) can modulate the functional status of fibroblasts in cancer. Here, we further delineated the role of sqstm 1 in PaSCs. The analysis of PDAC patient samples revealed reduction of sqstml expression in activated PaSCs in both mRNA and protein level. Downregulated sqstml via shRNA in PaSCs led to an inflammatory and senescent phenotype with increased IL8, CXCL1, and CXCL2 expression. Further analysis demonstrated that increased intracellular reactive oxygen species level contributed to the senescence in sqstm 1-downregulated PaSCs. This was mediated via impaired NRF2 activity since reduced sqstm 1 resulted in accumulation of KEAPI. Meanwhile, we found that sqstm 1 degradation caused by enhanced autophagy was not associated with transformation of senescent phenotype. At last, the data revealed that sqstml-downregulated PaSCs promoted pancreatic tumor cell growth, invasion, and macrophage phenotype transformation. Collectively, the current study indicated that sqstm l controlled transformation of senescent phenotype of PaSCs, which in turn is pro-tumorigenic.
\end{abstract}

Key words: sqstm1, pancreatic stellate cells, pancreatic adenocarcinoma, reactive oxygen species, senescence

\section{Introduction}

Pancreatic ductal adenocarcinoma (PDAC) is a lethal malignancy, projected to be the second leading cause of cancer-related death in western countries [1]. Treatment options are scarce for patients with advanced stage disease, and largely rely on modestly effective chemotherapies like gemcitabine alone or in combination with nab-paclitaxel, and FOLFIRINOX regimen $[2,3]$. A large number of trials with testing of targeted therapies have been disappointing in PDAC [4]. This is probably rooted from the genomic and molecular heterogeneity, and complicated tumor microenvironment as well. 
PDAC is characterized by a unique desmoplastic stroma consisting of activated fibroblasts, abundant extracellular matrix, and scarce blood vessels [5]. Pancreatic stellate cells (PaSCs) are a type of myofibroblast-like cell locating in the exocrine region of normal pancreas, and transformed from quiescent state to activated phenotype upon external stimuli during tumorigenesis. Mounting evidence indicated that activated PaSCs exerted pro-tumor effect through direct interaction with tumor cells, regulating angiogenesis and other constituents within microenvironment. However, recent studies uncovered an opposing outcome as shown by accelerated tumor progression when PaSCs were ablated in genetic engineering mouse models [6-8]. These paradoxical findings indicated a complex role and heterogeneity of PaSCs in pancreatic cancer, and further understanding of molecular mechanisms underlying PaSCs phenotype regulation are needed [9]. Indeed, several studies have indicated that subsets of PaSCs or cancer-associated fibroblasts (CAFs) existed with distinct function in tumor microenvironment [10-12]. This emphasized the importance of delineating molecular mechanisms regulating the specific PaSCs phenotypes.

PaSCs activation in PDAC is associated with complex cytokine released within microenvironment and tumor-stroma interaction [13]. Hedgehog signaling pathway has been viewed as a major contributor for PaSCs activation, wherein sonic hedgehog secreted from tumor cells as a ligand interacts with specific receptors on PaSCs $[14,15]$. In addition, other growth factors and cytokines like TGF- $\beta$, PDGF and interleukin- 6 cooperatively recruit and drive CAFs differentiation and proliferation. However, the intrinsic mechanisms of PaSCs activation are not fully appreciated $[16,17]$. Sequestosome-1 (sqstm1) has been recognized as an autophagic substrate and signaling adaptor that regulates many biological functions $[18,19]$. Studies have shown that CAFs had reduced sqstm1 expression in several types of cancer like prostate cancer and hepatocellular carcinoma (HCC), and downregulated sqstm1 in CAFs can promote cancer progression [20]. As pancreatic adenocarcinoma has a unique stromal feature compared with other cancer, it is attracting to determine whether sqstm1 also modulates pancreatic CAFs phenotype and function.

In the present study, we found that sqstm1 expression was remarkably lower in stromal cells than tumor cells, and the activation status of PaSCs correlated with sqstm1 level. Knockdown of sqstm1 led to pro-inflammatory and senescent phenotype of PaSCs through upregulation of ROS level. This phenotype in turn promoted pancreatic cancer progression.

\section{Materials and Methods}

\section{Cell lines and culture condition}

PaSCs line was purchased (ScienCell Research Laboratories, Carlsbad, CA) and cultured using Stellate Cell Medium. Pancreatic cancer cell lines Panc-1 and SW1990 was obtained from ATCC. Primary CAFs lines were generated from two resected PDAC fresh tissues, and their phenotype was confirmed by typical spindle-shape and immunostaining of a-SMA, vimentin, cytokeratin-19, and CD45. The primary CAFs used for experiment are within ten passages.

\section{Lentiviral vector production and infection of cells}

Small-hairpin RNA (shRNA) vector targeting human sqstm1 was purchased (Sigma-Aldrich), and the knockdown efficiency was validated by qPCR. Then the vector was packaged into lentivirus for further experiment. PaSCs were infected with lentiviral vector using appropriate multiplicity of infection (MOI), which was followed by selection with puromycin. The shRNA sequences targeting sqstm1 were: shRNA 1, TAGTACAACTGCTAGTTATTT; shRNA 2, CCCTTTGTCTTGTAGTTGCAT.

\section{Small interference RNA (siRNA) transfection}

SiRNA targeting human NRF2 was transfected into PaSCs with lipofectamine RNAiMAX (Thermo Fisher). The downstream analysis was performed after two days of transfection. The sequence used for siRNA is as follows: TTAAGACACTGTAACTCAGG AA.

\section{In vivo xenograft experiment}

Six-week-old female nude BALB/c mice (Shanghai SLAC Laboratory Animal Co., Ltd) were housed under pathogen-free conditions. $5 \times 10^{5}$ Panc-1 cells with equal number of PaSCs carrying NC or sqstm1-shRNA vector were injected subcutaneously ( $\mathrm{n}=5$ per group). Tumor growth were monitored by measurement of size every three days. Mice were euthanized after three weeks, and tumor specimens were collected for further analysis. All animal experiments were approved by the Second Affiliated Hospital of Zhejiang University Animal Care and Use Committee and were performed in accordance with their established guidelines.

\section{Immunohistochemistry and Immunofluorescence}

Human or mouse tumors were fixed in 10\% neutral-buffered formalin, embedded in paraffin, 
sectioned, stained with haematoxylin and eosin, and processed for immunostaining. Paraffin-embedded tissue sections were subjected to de-paraffinization and rehydration, and then were immersed in a pre-heated antigen retrieval water bath with a $\mathrm{pH} 6.1$ citrate buffer for $20 \mathrm{~min}$ at $95-96^{\circ} \mathrm{C}$. Tissue microarray of pancreatic cancer was purchased from Shanghai Outdo Biotech (Shanghai, China). For immunofluorescence, tissue sections were blocked with $1 \%$ goat serum or $3 \%$ BSA for $30 \mathrm{~min}$. Then slides were stained with primary antibodies overnight at $4^{\circ} \mathrm{C}$ followed by secondary antibodies staining. After washing, sections were covered with Vectashield Mounting Medium containing DAPI (Vector Laboratories, Burlingame, California, USA).

\section{Real-time PCR analysis}

Total RNA was extracted using TRIzol reagent. RNA was reverse transcribed using Superscript cDNA synthesis kit (Applied Biosystems). Then quantitative real-time PCR was performed with STBR Green RT-PCR kit (Bio-Rad Laboratories) on QuantStudio PCR system (Applied Biosystems). The relative expression of target genes was measured in triplicates, and calculated by comparative CT method

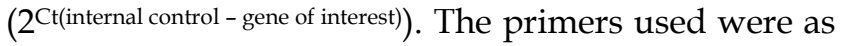
follows: SQSTM1 F: GCCAGAGGAACAGATGGA GT, SQSTM1 R: TCCGATTCTG GCATCTGTAG; a-SMA F: ACTGAGCGTGGCTATTCCTCCGTT, R: GCAGTGGCCATCTCATTTTCA; NRF2 F: GCGACG GAAAGAGTATGAGC, NRF2 R: ACGTAGCCGAAG AAACCTCA; NQO1 F: GCCGAGTCTGTTCTGGCT TA, NQO1 R: CTGCCTTCTTACTCCGGAAGG; HO-1 F: TTCAAGCAGCTCTACCGCTC, HO-1 R: GGGGG CAGAATCTTGCACTT; GCLC F: TCCAGGTGACAT TCCAAGCC GCLC R: AGATGCAGCACTCAAAG CCA; $\beta$-actin F: CTGGGTATGGAATCCTGTGG, R: GTACTTGCGCTCAGGAGGAG; GAPDH F: GCCAA GGTCATCCATGACAACT, R: GAGGGGCCATCCA CAGTCT.

\section{Immunoblotting}

Cells were lysed in ice-cold RIPA buffer supplemented with proteinase inhibitor. Protein concentration of cell lysates was quantified using BCA method. 50ug protein was loaded on $10 \%$ Bis-Tris gel and separated by SDS-PAGE. Then it was transferred onto PVDF membranes. The following antibodies were used for immunoblotting: anti-GAPDH (CST; \#5174), anti-Sqstm1 (Abcam; ab155686), anti-a-SMA (Abcam; ab124964), anti-Keap1 (CST; \#8047), anti-Nrf2 (CST; \#12721), anti-Fibronectin (Abcam; ab32419), anti-LC3 (CST; \#4108).

\section{ROS measurement}

ROS in cultured cells was measured by incubating $2 \times 10^{5}$ PaSCs with $5 \mathrm{uM} \mathrm{CM}-\mathrm{H}_{2}$ DCFDA (Life technologies) for $30 \mathrm{~min}$ at $37^{\circ} \mathrm{C}$. The fluorescence was detected via flow cytometry and analyzed by FlowJo software (Tree Star).

\section{Fluorescence activated cell sorting (FACS)}

For fresh primary pancreatic tumor tissue, single cell suspension was made by mechanical and enzymatic dissociation. Cells were blocked with Fc blocking reagent (BD Bioscience). After labeling of Live/Dead dye, cells were stained with EpCAM-PE (Biolegend) and FAP-APC (R\&D) on ice for $30 \mathrm{~min}$ for sorting (BD Aria II). The gating was based on the isotype staining.

\section{$\boldsymbol{\beta}$-galactosidase staining}

Cell senescence was evaluated by $\beta$-galactosidase staining (CST) following manufacturer's instructions. Briefly, $1 \times 10^{5}$ PaSCs were seeded onto 4 -well slide chamber and fixed by $4 \%$ paraformaldehyde the next day. Cells were stained with $\beta$-galactosidase solution and incubated overnight in a dry incubator without carbon dioxide. The senescent cells with blue color were counted under microscope the next day.

\section{Statistical analysis}

Data were presented as means \pm s.d. Unpaired student $t$ test was used for comparison of continuous parameters. All statistical calculations were performed using Prism 7.0 (Graphpad, La Jolla, CA). Two-sized $p$ value less than 0.05 was considered as significant.

\section{Results}

\section{Distinct sqstm 1 expression status in tumor and stromal compartment of PDAC}

Sqstm1 expression in PDAC was firstly determined. The immunohistochemistry for tissue microarray consisting of fifty pancreatic cancer tissues revealed that sqstm1 was predominantly present in tumor cells rather than stroma in most patients (Figure 1A). Both nuclear and cytoplasmic staining for sqstm1 were observed in stromal and tumor cells. The immunofluorescence in two cases confirmed markedly lower abundance of sqstm1 in a-SMA positive stroma in contrast to tumor compartment (Figure 1B). Then we examined the LC3 expression in tumor tissues as sqstm1 protein abundance is tightly controlled by cell autophagic level. The result showed $\mathrm{LC}^{+}$puncta within stromal cells in over half of the cases (29/50) (Figure 1A). This indicated that active autophagy might contribute to the low sqstm1 protein abundance in a portion of patients. 
A

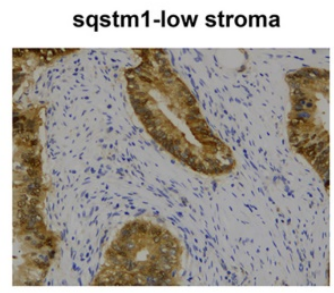

sqstm1-high stroma

LC3

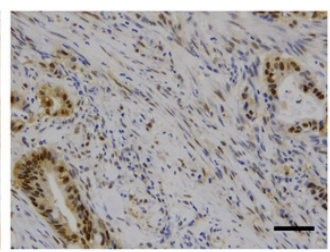

\begin{tabular}{lcc}
\hline & sqstm1-low & sqstm1-high \\
\hline Tumor & 5 & 45 \\
Stroma & 38 & 12 \\
\hline
\end{tabular}

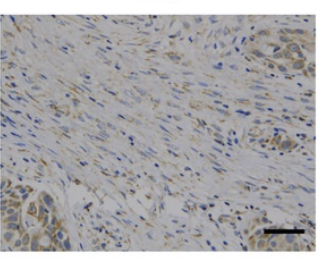

B

Sqstm1

a-SMA

Merge

C

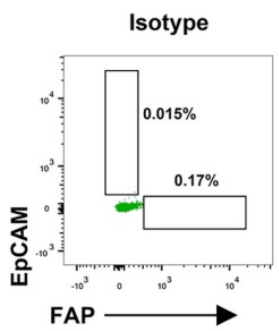

EpCAM/FAP
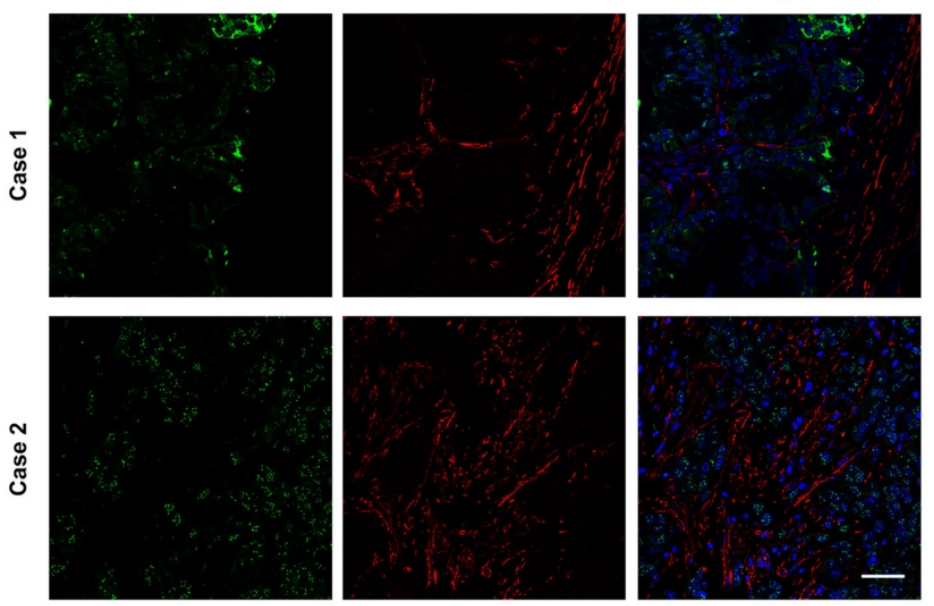

FAP

E

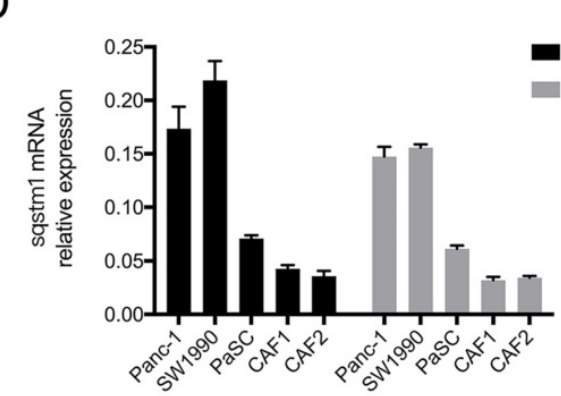

-actin as reference GAPDH as reference

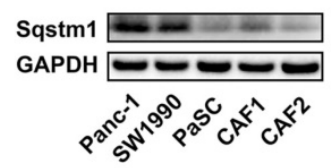

F

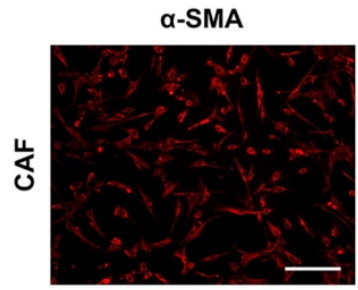

G

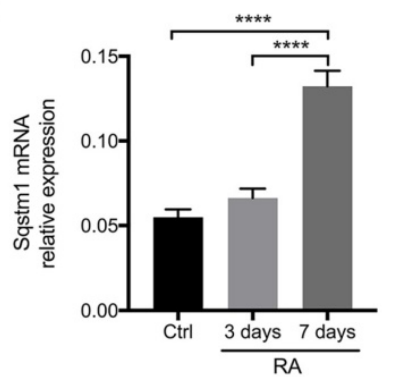

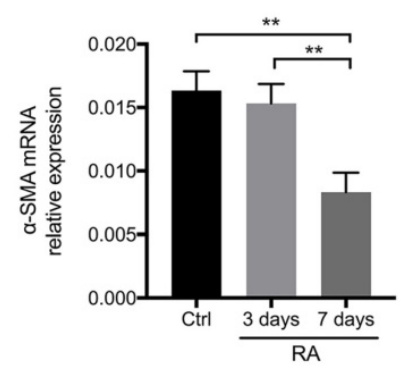

$\mathrm{H}$

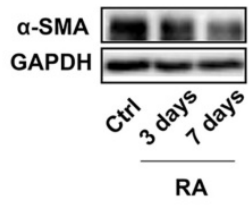

Figure 1. Sqstml expression was downregulated in cancer-associated fibroblasts (CAFs) of pancreatic cancer. (A) Representative images of immunohistochemistry (IHC) staining for sqstm 1 and LC3 in pancreatic cancer tissues. The table shows expression patterns of sqstm 1 in tumor and stromal compartment based on IHC staining. Scale bars represent $50 \mu \mathrm{m}$. (B) Representative images of double immunofluorescence staining for sqstml and $\alpha$-SMA in pancreatic cancer tissues. Scale bars represent $50 \mu \mathrm{m}$. (C) Fluorescence activated cell sorting (FACS) for EpCAM+ and FAP ${ }^{+}$cells and qPCR examining sqstml mRNA level for sorted cells. ${ }^{*} p<0.05$. (D) Examination of sqstm 1 mRNA expression by qPCR for pancreatic cell line and cultured primary CAFs line using $\beta$-actin and GAPDH as internal control, respectively. (E) Western blot analysis for sqstml for pancreatic cell line and cultured primary CAFs line. (F) Representative image of immunofluorescence staining for $\alpha$-SMA in one cultured CAFs line was showed. Scale bars represent $20 \mu \mathrm{m}$. (G) qPCR for sqstml in pancreatic stellate cells (PaSCs) following retinoid acid treatment. (H) Western blot analysis for $\alpha-S M A$ in PaSCs following retinoid acid treatment. ${ }^{* *} p<0.01$, ${ }^{* * * * *} p<0.0001$.

To determine whether sqstm1 was transcriptionally reduced in CAFs, we sorted EpCAM+ tumor cells and $\mathrm{FAP}^{+} \mathrm{CAFs}$ from three fresh pancreatic cancer tissues. The qPCR result confirmed that sqstm1 mRNA was remarkably lower in CAFs than epithelial tumor cells (Figure 1C). In addition, primary CAFs 
lines were successfully established from two resected pancreatic cancer specimens. Compared with PDAC cell line Panc-1 and Miapaca2, the primary CAFs exhibited decreased sqstm1 mRNA expression (Figure 1D). The lower sqstm1 expression was also confirmed by western blot (Figure 1E). The phenotype of primary CAFs was corroborated by positive a-SMA staining (Figure $1 \mathrm{~F}$ ). Of note, an in vitro cultured PaSCs line also had relatively lower sqstm1 mRNA level (Figure 1E-F). It has been shown that retinoid acid can induce reversion of activated fibroblasts to quiescent phenotype [21-23]. Thus we analyzed the change of sqstm1 expression under quiescence induction. Our data confirmed that treatment with RA for 7 days led to reduction of a-SMA expression (Figure 1G-H). In parallel, sqstm1 mRNA expression was significantly increased (Figure 1G). Taken together, these data indicated that sqstm1 was downregulated in activated PaSCs.

\section{Sqstm I modulated pro-inflammatory response in PaSCs}

We next explored the molecular consequence of downregulated sqstm1 on PaSCs. Two different shRNA targeting sqstm1 was transfected into PaSCs line, and the downregulation of sqstm 1 was validated by western blot (Figure 2A). The microarray data revealed over 400 differentially expressed genes between PaSCs with transfection of either negative control (sqstm1-NC PaSCs) or sqstm1 shRNA vector (sqstm1-shRNA PaSCs). Among the selected genes with over 2-fold change, expression for several inflammatory factors was significantly elevated in
shRNA-treated cells (Figure 2B). qPCR analysis corroborated the upregulation of these genes including IL-8, CXCL1, CXCL2, CCL2, and CCL20 (Figure 2C). This transcriptional change suggested an inflammatory and senescent phenotype of PaSCs. Flow cytometry analysis showed that FAP expression was increased in sqstm1 shRNA-transfected PaSCs (Figure 2D). Consistently, $\beta$-galactosidase staining demonstrated that downregulation of sqstm1 in PaSCs led to enhanced senescence (Figure 2E).

\section{Enhanced autophagy did not contribute to the pro-inflammatory and senescent phenotype of PaSCs}

Sqstm1 abundance is tightly controlled by autophagic degradation, and thus we determined whether autophagy also modulates inflammatory phenotype transformation in PaSCs. Autophagy was induced by starvation or rapamycin treatment, and western blot confirmed increased LC3II to LC3I ratio and sqstm1 downregulation in both conditions (Figure 3A). The induction of autophagy facilitated activation of PaSCs as shown by upregulated a-SMA and fibronectin expression (Figure 3A). Nevertheless, there was no change in IL-8, CXCL1, CXCL2 expression after induction of autophagy (Figure 3B). Furthermore, comparable senescence level was observed for PaSCs following starvation or rapamycin treatment (Figure 3C). This suggested that autophagy did not fully recapitulate the functional effects of transcriptional downregulation of sqstm1 in PaSCs.
A

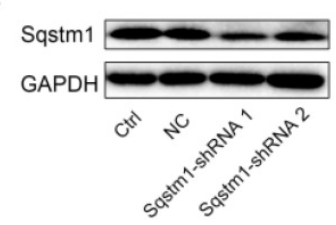

B

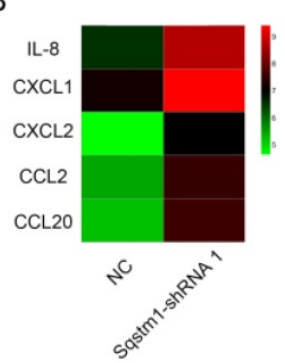

D
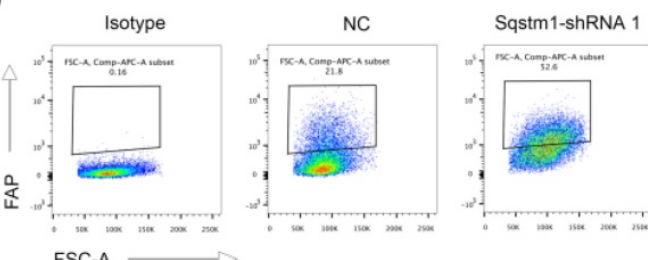

C
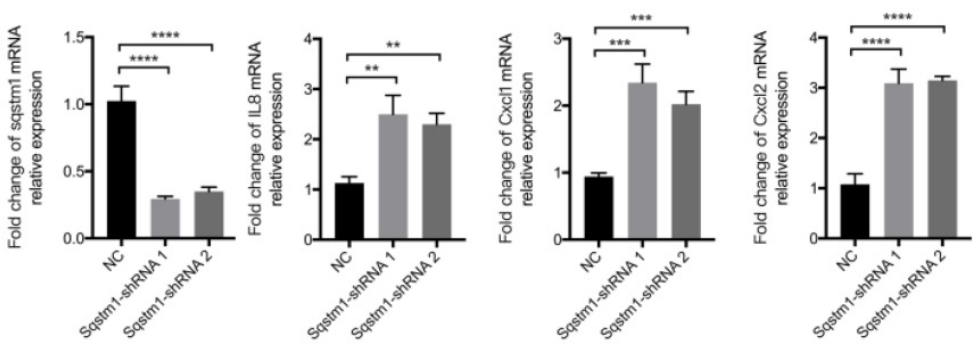

E

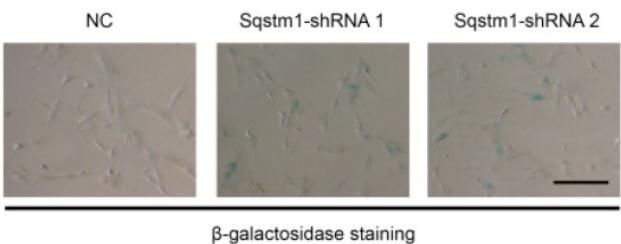

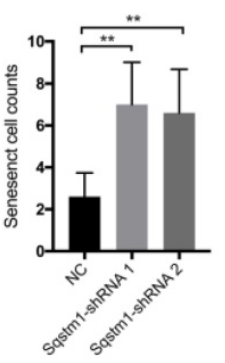

Figure 2. Sqstm1 downregulation induces inflammatory and senescent phenotype of PaSCs. (A) Validation of sqstm1 downregulation following shRNA transfection by western blot in PaSCs. (B) Gene microarray for comparison of transcriptome between negative control and shRNA-transfected PaSCs. (C) Validation of expression change for IL8, CXCL1, and CXCL2 by qPCR. (D) Flow cytometry analysis for FAP expression in negative control and shRNA-transfected PaSCs. (E) $\beta$-galactosidase staining for senescence analysis. ${ }^{* *} p<0.01,{ }^{* * *} p<0.001$. 
A

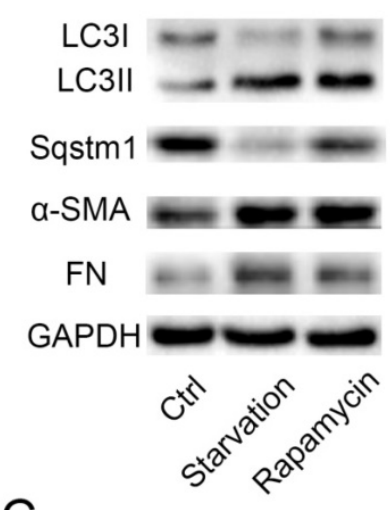

B

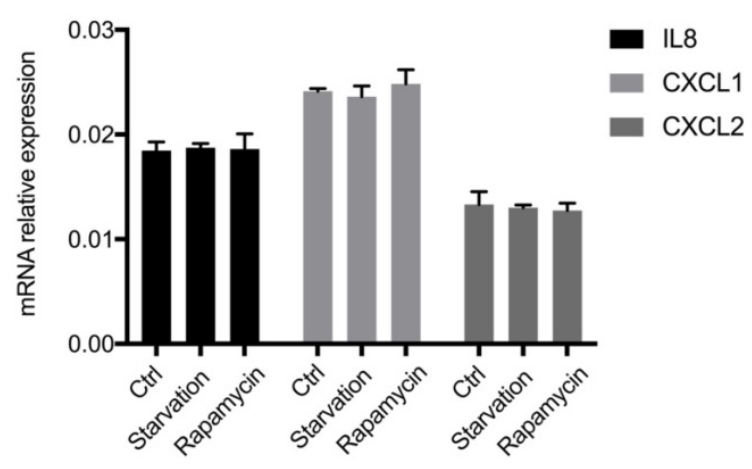

C

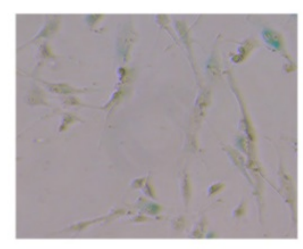

Ctrl

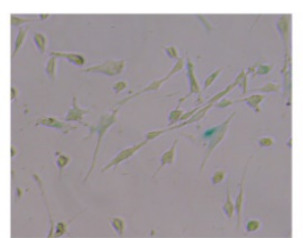

Starvation

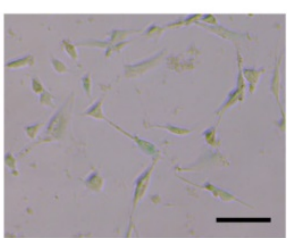

Rapamycin

Figure 3. Autophagy induces PaSCs activation but not senescence. (A) Western blot analysis for markers of autophagy and fibroblasts activation in PaSCs upon autophagy induction. (B) qPCR analysis for IL8, CXCL1, and CXCL2 in PaSCs upon autophagy induction. (C) $\beta$-galactosidase staining for PaSCs upon starvation or rapamycin treatment. Scale bar represents $20 \mu \mathrm{c}$.

\section{Enhanced ROS production mediated the inflammatory phenotype transformation upon sqstm 1 reduction}

The mechanisms for regulation of inflammatory phenotype by sqstm 1 was then investigated. We analyzed the reactive oxygen species (ROS) level in PaSCs as signaling adaptor sqstm1 has crucial function in modulating ROS production. As expected, PaSCs with reduction of sqstm 1 had markedly higher ROS level compared with control group (Figure 4A). NAC, a well-characterized anti-oxidant, inhibited ROS generation in sqstm1 shRNA-transfected PaSCs. Meanwhile, it led to downregulation of inflammatory factor IL-8, CXCL1, and CXCL2 expression (Figure $4 \mathrm{~B})$. Further analysis revealed that KEAP1 protein abundance increased along with decreased NRF2 level, suggesting that autophagic degradation of KEAP1 via sqstm1 was inhibited (Figure $4 \mathrm{C}$ ). The immunofluorescence staining showed that nuclear accumulation of NRF2 was remarkably blocked upon sqstm1 downregulation (Figure 4D). QPCR analysis demonstrated that sqstm1 shRNA-transfected PaSCs could significantly downregulate expression of NRF2-targets NADPH quinone oxidoreductase 1 (Nqo1), Heme oxygenase-1 (HO-1), and Glutamatecysteine ligase, catalytic (Gclc) compared with negative control (Figure 4E). NRF2 has been wellcharacterized as a transcription factor in promoting anti-oxidative response, and its decrease could be responsible for the enhanced ROS production.
Furthermore, downregulation of NRF2 expression by RNA interference led to enhanced PaSCs senescence (Figure 4F-G).

\section{Regulation of sqstm 1 in PaSCs had pleiotropic effects on pancreatic tumor cells}

The impact of transformed PaSCs phenotype caused by sqstm1 downregulation on pancreatic cancer was evaluated through in vitro and in vivo studies. CCK-8 assay showed that conditioned medium from sqstm1-KD PaSCs accelerated Panc-1 cell proliferation compared with sqstm1-NC PaSCs (Figure 5A). The supernatant from sqstm1-KD PaSCs also significantly enhanced Panc- 1 cell invasion in transwell chamber assay (Figure 5B). Treatment with conditioned medium derived from sqstm1-KD PaSCs resulted in much higher CD206 expression than those from sqstm1-NC PaSCs (Figure 5C). The mouse experiment using pancreas orthotopic model demonstrated that co-injection of Panc-1 with sqstm1-KD PaSCs promoted tumor growth and liver metastasis (Figure 5D). Tumors in sqstm1-KD PaSCs group contained more a-SMA ${ }^{+}$fibroblasts and $\mathrm{Ki}^{+} 7^{+}$tumor cells (Figure 5E). In addition, as downregulation of sqstm1 in PaSCs was associated with increased CCL2 and CCL20 level, we then determined its effect on macrophage phenotype. Altogether, these data indicated that PaSCs with sqstm1 downregulation promoted tumor progression through multiple effects. 
A

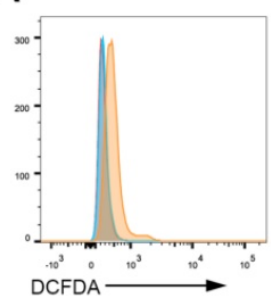

C

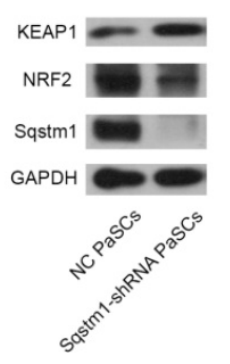

F

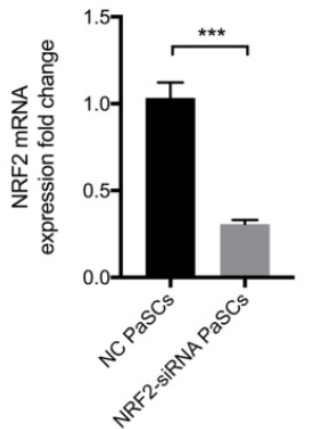

B
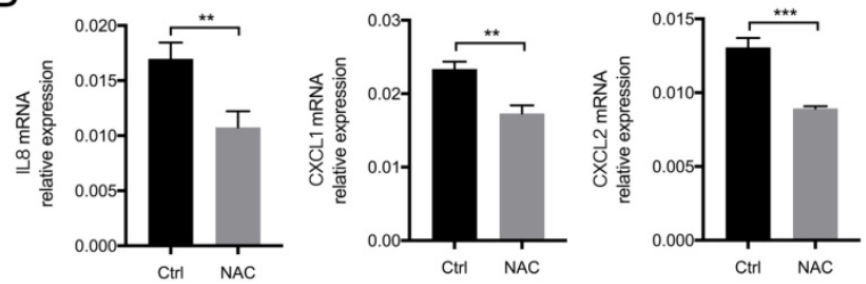

E
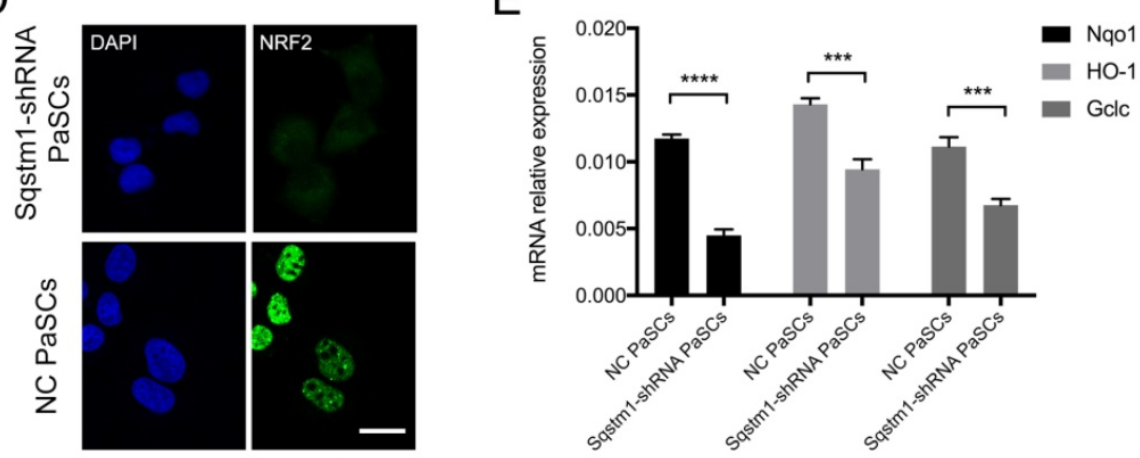

G

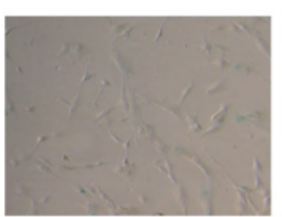

NC PaSCs

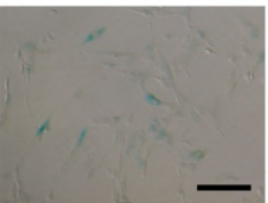

NRF2-siRNA PaSCs

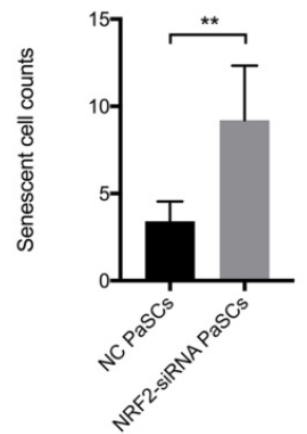

Figure 4. Sqstm1 regulates ROS balance through KEAPI/NRF2 signaling. (A) ROS measurement via DCFDA probe detection. (B) Examination of IL8, CXCLI, and CXCL2 mRNA level upon NAC treatment in PaSCs. (C) Western blot analysis for KEAP1 and NRF2 in sqstm 1-shRNA transfected PaSCs. (D) Immunofluorescence for NRF2 in sqstm1-downregulated PaSCs. Scale bar represents $10 \mu \mathrm{m}$. (E) qPCR for Nqol between negative control and sqstml-shRNA transfected PaSCs. (F) Validation of NRF2 expression change upon siRNA transfection in PaSCs by qPCR. (G) $\beta$-galactosidase staining for PaSCs upon transfection of NRF2-siRNA. Scale bar represents $20 \mu \mathrm{m} .{ }^{* *} p<0.01,{ }^{* * * *} p<0.001,{ }^{* * *+* k} p<0.0001$.

\section{Discussion}

Pancreatic cancer progresses with sequential acquisition of specific genetic alterations, including KRAS activating mutation as initiating event followed by loss-of-function in tumor suppressor genes like TP53, CDKN2A, and SMAD4 [24]. During this process, surrounding non-tumor cells evolve in parallel, and its crosstalk with tumor cells also contribute to the malignant transformation. Among them, PaSCs gradually differentiated into activated fibroblasts and proliferated, becoming the dominant component within tumor microenvironment. Previous studies have been largely focusing on the exogenous stimuli to PaSCs such as tumor cell-secreted hedgehog ligand and other growth factors. However, the endogenous regulation signaling is poorly understood.
In the current study, we demonstrated that sqstm1-Keap1/Nrf2-ROS cascade regulated proinflammatory and senescent response of PaSCs, and the resultant phenotype promoted tumor progression. Tumor microenvironment for PDAC is characterized with limited blood supply and inadequate nutrient resources, leading to extensive metabolic and oxidative stress on cells. It has been shown that pancreatic tumor cells could overcome these challenges through alternative metabolic machinery and anti-oxidative mechanisms due to oncogenic KRAS alteration and other epigenetic regulation [24]. In contrast, stromal cells are less resistant, and thus their phenotypes are more easily to be altered upon these stress. Recent findings indicated that senescence occurred in a subpopulation of pancreatic CAFs, and this subset contributed to tumor invasion and 
metastasis [25]. However, the underlying mechanisms are not fully understood. Many evidence has suggested that oxidative stress is associated with development of senescence both in tumor and non-tumor cells. Our data confirmed that upregulated ROS production resulted in increased number of senescent PaSCs. The balance of redox state within cells is delicately maintained. Intracellular ROS is produced upon various stimulations, and detoxification machinery is initiated to counteract the excess of oxidative derivatives. The anti-oxidative response is critical in that accumulated ROS can eventually disrupt cell homeostasis and lead to senescence and cell death. Our results indicated that sqstm1-mediated modulation of NRF2 abundance was critical for maintaining ROS balance in PaSCs. NRF2 is a well-characterized transcription factor to detoxify ROS products [26]. Its functional activity is directly controlled by Keap1 that can block the nuclear import of NRF2. As a autophagic substrate, sqstm1 could recruit Keap1 into autophagosome for degradation, and thus led to increased NRF2 nuclear accumulation. Of note, one study in prostate cancer revealed that increased ROS release in sqstm1deficient CAFs was due to metabolic reprogramming rather than NRF2-mediated effect[20]. As CAFs in pancreatic cancer are specifically derived PaSCs that is distinct from other types of cancer, it is possible that distinct mechanism involves in regulation of ROS balance. In fact, another study confirmed that NRF2 modulated ROS level in synovial fibroblasts[27].

A large number of evidence has revealed that sqstm1 is critical in tumor initiation and progression.
Many studies found overexpression of sqstm1 in cancer cells. It was showed that sqstm1 was sufficient to induce HCC in mice, and its high expression in hepatocytes adjacent to tumor predicted rapid recurrence after surgical resection [28]. Recent study suggested sqstm 1 accumulation in pancreatic ductal epithelium drove neoplastic transformation [29]. On the other hand, however, sqstm 1 can modulate the activity of stromal non-tumor cells like fibroblasts [30]. Unlike overexpression in tumor cells, sqstm1 abundance is largely downregulated in CAFs of various types of cancer. The immunostaining of PDAC tissues in the current study indicated a similar pattern of sqstm1 expression between tumor and stromal compartments. The reduced sqstm1 mRNA expression suggested inhibition at transcriptional level. Besides, it should be noted that the abundance of sqstm1 protein is under strict control of autophagy activity. Previous study demonstrated that pancreatic tumor cells induced autophagy in CAFs to secrete nutrient resources including non-essential amino acids [31]. Another finding suggested upregulated autophagy in CAFs for a significant proportion of PDAC patients and this phenotype of fibroblasts promoted tumor growth and invasion [32]. Indeed, we noted LC3 plaque within stromal compartment for over half of patients. Thus it is probably that sqstm1 level is regulated both transcriptionally and posttranscriptionally. Nevertheless, enhanced autophagy did not induce senescent phenotype in spite of downregulated sqstm1 protein level. This could be attributed to the complex effects triggered by autophagy besides the downregulation of sqstm1.
A

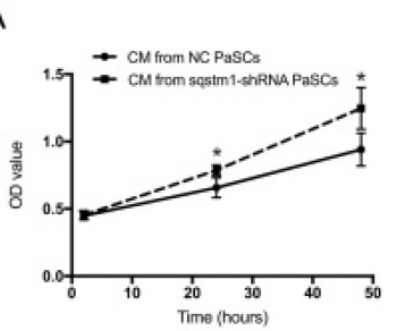

D

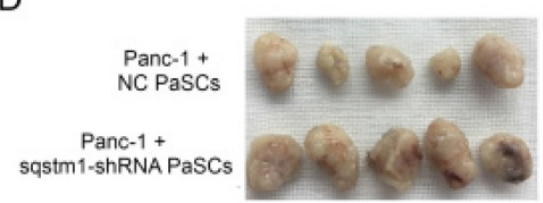

B

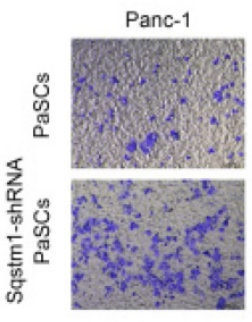

$E$
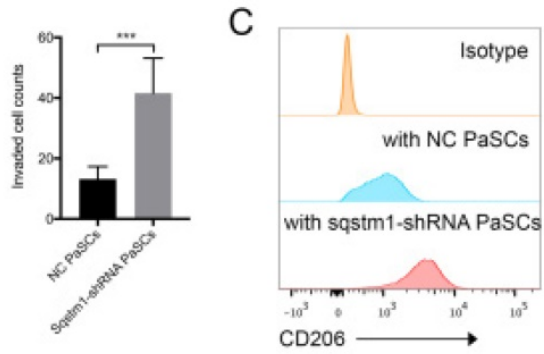

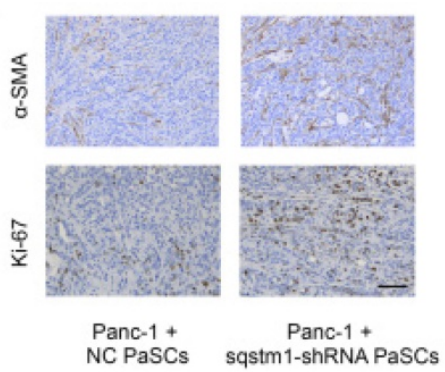

Figure 5. Sqstm 1-downregulated PaSCs promotes tumor growth and progression. Comparison of proliferation (A) and invasion (B) of Panc-1 cells upon treatment with conditioned medium (CM) from PaSCs with or without sqstm1 shRNA transfection. (C) Comparison of CD206 expression in monocytes treatment with CM from PaSCs with or without sqstm1 shRNA transfection. (D-E) In vivo tumor xenograft of Panc-1 co-injected with PaSCs or sqstml-shRNA transfected PaSCs. IHC staining for $\alpha-S M A$ and Ki-67 was analyzed. ${ }^{*} p<0.05$, ${ }^{* * * *} p<0.001$. 
Senescent cells typically show elevated expression of multiple inflammatory factors. Thus, the consequent pro-tumorigenic role exerted by senescent PaSCs is probably caused by the complex effect of paracrine signaling. IL6 is a well-characterized inflammatory factor with pro-tumorigenic function [33]. Its secretion is significantly upregulated in prostate CAFs with sqstm1 knockdown, which is corroborated in the present study for PaSCs. A recent finding indicated that CAFs undergoing senescence enhanced tumor cells metastasis in a IL8-dependent manner [25]. In fact, IL8 is among the most upregulated genes in sqstm1-knockdown PaSCs. In addition, we also found that PaSCs with reduced sqstm1 expression promoted differentiation of macrophages to pro-tumor M2 phenotype. CCL2 and CCL20 expression were increased in sqstm1knockdown PaSCs, and these two cytokines have been shown to induce monocytes recruitment and differentiation [34].

In conclusion, our data supports that sqstm1/ NRF2/ROS modulates senescent phenotype of PaSCs, which in turn promotes pancreatic tumor cell progression. This suggests that pancreatic tumor and stromal cells differentially expressed sqstm1 and cooperatively facilitate tumor progression.

\section{Acknowledgement}

This work was financially supported by Projects of Medical and Health Technology Plan Program in Zhejiang Province (2015KYB454), Projects of Lishui Key Research and Development Plan in Zhejiang Province (2016ZDYF01, 2017ZDYF12), the important subject fund of Zhejiang province science and technology hall (2015C03033) and National Natural Science Foundation of China (81302071, 81673809 and 81573953).

\section{Competing Interests}

The authors have declared that no competing interest exists.

\section{References}

1. Rahib L, Smith BD, Aizenberg R, Rosenzweig AB, Fleshman JM, Matrisian LM. Projecting cancer incidence and deaths to 2030: the unexpected burden of thyroid, liver, and pancreas cancers in the United States. Cancer research. 2014; 74: 2913-21.

2. Conroy T, Desseigne F, Ychou M, Bouche O, Guimbaud R, Becouarn Y, et al. FOLFIRINOX versus gemcitabine for metastatic pancreatic cancer. The New England journal of medicine. 2011; 364: 1817-25.

3. Von Hoff DD, Ervin T, Arena FP, Chiorean EG, Infante J, Moore M, et al. Increased survival in pancreatic cancer with nab-paclitaxel plus gemcitabine. The New England journal of medicine. 2013; 369: 1691-703.

4. Chiaravalli M, Reni M, O'Reilly EM. Pancreatic ductal adenocarcinoma: State-of-the-art 2017 and new therapeutic strategies. Cancer treatment reviews. 2017; 60: 32-43.

5. Neesse A, Algul H, Tuveson DA, Gress TM. Stromal biology and therapy in pancreatic cancer: a changing paradigm. Gut. 2015; 64: 1476-84.

6. Ozdemir BC, Pentcheva-Hoang T, Carstens JL, Zheng X, Wu CC, Simpson TR, et al. Depletion of carcinoma-associated fibroblasts and fibrosis induces immunosuppression and accelerates pancreas cancer with reduced survival. Cancer cell. 2014; 25: 719-34.

7. Rhim AD, Oberstein PE, Thomas DH, Mirek ET, Palermo CF, Sastra SA, et al. Stromal elements act to restrain, rather than support, pancreatic ductal adenocarcinoma. Cancer cell. 2014; 25: 735-47.

8. Lee JJ, Perera RM, Wang H, Wu DC, Liu XS, Han S, et al. Stromal response to Hedgehog signaling restrains pancreatic cancer progression. Proceedings of the National Academy of Sciences of the United States of America. 2014; 111: E3091-100.

9. Sherman MH, Yu RT, Engle DD, Ding N, Atkins AR, Tiriac H, et al. Vitamin D receptor-mediated stromal reprogramming suppresses pancreatitis and enhances pancreatic cancer therapy. Cell. 2014; 159: 80-93.

10. Waghray $M$, Yalamanchili $M$, Dziubinski M, Zeinali M, Erkkinen M, Yang H, et al. GM-CSF Mediates Mesenchymal-Epithelial Cross-talk in Pancreatic Cancer. Cancer discovery. 2016; 6: 886-99.

11. Ohlund D, Handly-Santana A, Biffi G, Elyada E, Almeida AS, Ponz-Sarvise M, et al. Distinct populations of inflammatory fibroblasts and myofibroblasts in pancreatic cancer. The Journal of experimental medicine. 2017; 214: 579-96.

12. Ohlund D, Elyada E, Tuveson D. Fibroblast heterogeneity in the cancer wound. The Journal of experimental medicine. 2014; 211: 1503-23.

13. Thayer SP, di Magliano MP, Heiser PW, Nielsen CM, Roberts DI, Lauwers GY, et al. Hedgehog is an early and late mediator of pancreatic cancer tumorigenesis. Nature. 2003; 425: 851-6.

14. Bailey JM, Swanson BJ, Hamada T, Eggers JP, Singh PK, Caffery T, et al. Sonic hedgehog promotes desmoplasia in pancreatic cancer. Clinical cancer research : an official journal of the American Association for Cancer Research. 2008; 14 : 5995-6004.

15. Fendrich V, Oh E, Bang S, Karikari C, Ottenhof N, Bisht S, et al. Ectopic overexpression of Sonic Hedgehog (Shh) induces stromal expansion and metaplasia in the adult murine pancreas. Neoplasia (New York, NY). 2011; 13: 923-30.

16. Kang N, Shah VH, Urrutia R. Membrane-to-Nucleus Signals and Epigenetic Mechanisms for Myofibroblastic Activation and Desmoplastic Stroma: Potential Therapeutic Targets for Liver Metastasis? Molecular cancer research : MCR. 2015; 13: 604-12

17. Xu Y, Zhou X, Mei M, Ren Y. Reprograming carcinoma associated fibroblasts by microRNAs. Current molecular medicine. 2017.

18. Komatsu M, Ichimura Y. Physiological significance of selective degradation of p62 by autophagy. FEBS letters. 2010; 584: 1374-8.

19. Moscat J, Karin M, Diaz-Meco MT. p62 in Cancer: Signaling Adaptor Beyond Autophagy. Cell. 2016; 167: 606-9.

20. Valencia T, Kim JY, Abu-Baker S, Moscat-Pardos J, Ahn CS, Reina-Campos M, et al. Metabolic reprogramming of stromal fibroblasts through p62-mTORC1 signaling promotes inflammation and tumorigenesis. Cancer Cell. 2014; 26: 121-35.

21. Froeling FE, Feig C, Chelala C, Dobson R, Mein CE, Tuveson DA, et al. Retinoic acid-induced pancreatic stellate cell quiescence reduces paracrine Wnt-beta-catenin signaling to slow tumor progression. Gastroenterology. 2011; 141: 1486-97, 97 e1-14.

22. McCarroll JA, Phillips PA, Santucci N, Pirola RC, Wilson JS, Apte MV. Vitamin A inhibits pancreatic stellate cell activation: implications for treatment of pancreatic fibrosis. Gut. 2006; 55: 79-89.

23. Chronopoulos A, Robinson B, Sarper M, Cortes E, Auernheimer V, Lachowski D, et al. ATRA mechanically reprograms pancreatic stellate cells to suppress matrix remodelling and inhibit cancer cell invasion. Nat Commun. 2016; 7: 12630.

24. Bailey P, Chang DK, Nones K, Johns AL, Patch AM, Gingras MC, et al. Genomic analyses identify molecular subtypes of pancreatic cancer. Nature. 2016; 531: 47-52

25. Wang T, Notta F, Navab R, Joseph J, Ibrahimov E, Xu J, et al. Senescent Carcinoma-Associated Fibroblasts Upregulate IL8 to Enhance Prometastatic Phenotypes. Molecular cancer research : MCR. 2017; 15: 3-14.

26. DeNicola GM, Karreth FA, Humpton TJ, Gopinathan A, Wei C, Frese K, et al. Oncogene-induced Nrf2 transcription promotes ROS detoxification and tumorigenesis. Nature. 2011; 475: 106-9.

27. Su X, Huang Q, Chen J, Wang M, Pan H, Wang R, et al. Calycosin suppresses expression of pro-inflammatory cytokines via the activation of p62/Nrf2-linked heme oxygenase 1 in rheumatoid arthritis synovial fibroblasts. Pharmacol Res. 2016; 113: 695-704.

28. Umemura A, He F, Taniguchi K, Nakagawa H, Yamachika S, Font-Burgada J, et al. p62, Upregulated during Preneoplasia, Induces Hepatocellular Carcinogenesis by Maintaining Survival of Stressed HCC-Initiating Cells. Cancer cell. 2016; 29: 935-48.

29. Todoric J, Antonucci L, Di Caro G, Li N, Wu X, Lytle NK, et al. Stress-Activated NRF2-MDM2 Cascade Controls Neoplastic Progression in Pancreas. Cancer cell. 2017.

30. Duran A, Hernandez ED, Reina-Campos M, Castilla EA, Subramaniam S, Raghunandan S, et al. p62/SQSTM1 by Binding to Vitamin D Receptor Inhibits Hepatic Stellate Cell Activity, Fibrosis, and Liver Cancer. Cancer cell. 2016; 30: 595-609.

31. Sousa CM, Biancur DE, Wang X, Halbrook CJ, Sherman MH, Zhang L, et al. Pancreatic stellate cells support tumour metabolism through autophagic alanine secretion. Nature. 2016; 536: 479-83.

32. Endo S, Nakata K, Ohuchida K, Takesue S, Nakayama H, Abe T, et al. Autophagy Is Required for Activation of Pancreatic Stellate Cells, Associated 
With Pancreatic Cancer Progression and Promotes Growth of Pancreatic Tumors in Mice. Gastroenterology. 2017; 152: 1492-506.e24.

33. Wu YS, Chung I, Wong WF, Masamune A, Sim MS, Looi CY. Paracrine IL-6 signaling mediates the effects of pancreatic stellate cells on epithelial-mesenchymal transition via Stat3/Nrf2 pathway in pancreatic cancer cells. Biochimica et biophysica acta. 2017; 1861: 296-306.

34. Geismann C, Grohmann F, Dreher A, Hasler R, Rosenstiel P, Legler K, et al.

Role of CCL20 mediated immune cell recruitment in NF-kappaB mediated TRAIL resistance of pancreatic cancer. Biochimica et biophysica acta. 2017; 1864: 782-96. 